\title{
Demonstration of acoustic waveguiding and tight bending in phononic crystals
}

M. Ghasemi Baboly, A. Raza, J. Brady, C. M. Reinke, Z. C. Leseman, and I. El-Kady’

Citation: Appl. Phys. Lett. 109, 183504 (2016); doi: 10.1063/1.4966463

View online: $h t t p: / / d x . d o i . o r g / 10.1063 / 1.4966463$

View Table of Contents: http://aip.scitation.org/toc/apl/109/18

Published by the American Institute of Physics

\section{Articles you may be interested in}

Planar ring-shaped phononic crystal anchoring boundaries for enhancing the quality factor of Lamb mode resonators

Applied Physics Letters 109, 203501 (2016); 10.1063/1.4967794

Ultra-high frequency, high Q/volume micromechanical resonators in a planar AIN phononic crystal

Journal of Applied Physics 120, 034502 (2016); 10.1063/1.4958671

Experimental investigation of energy localization in line-defect resonator based on silicon locally resonant phononic crystal

Applied Physics Letters 109, 161102 (2016); 10.1063/1.4963816

Focusing of ultrasonic waves by negative refraction in phononic crystals

AIP Advances 6, 121606 (2016); 10.1063/1.4972204

High- $Q$ micromechanical resonators in a two-dimensional phononic crystal slab

Applied Physics Letters 94, 051906 (2009); 10.1063/1.3078284

Micro-silicon phononic crystal with locally resonant theory

Applied Physics Letters 110, 171902 (2017); 10.1063/1.4981121

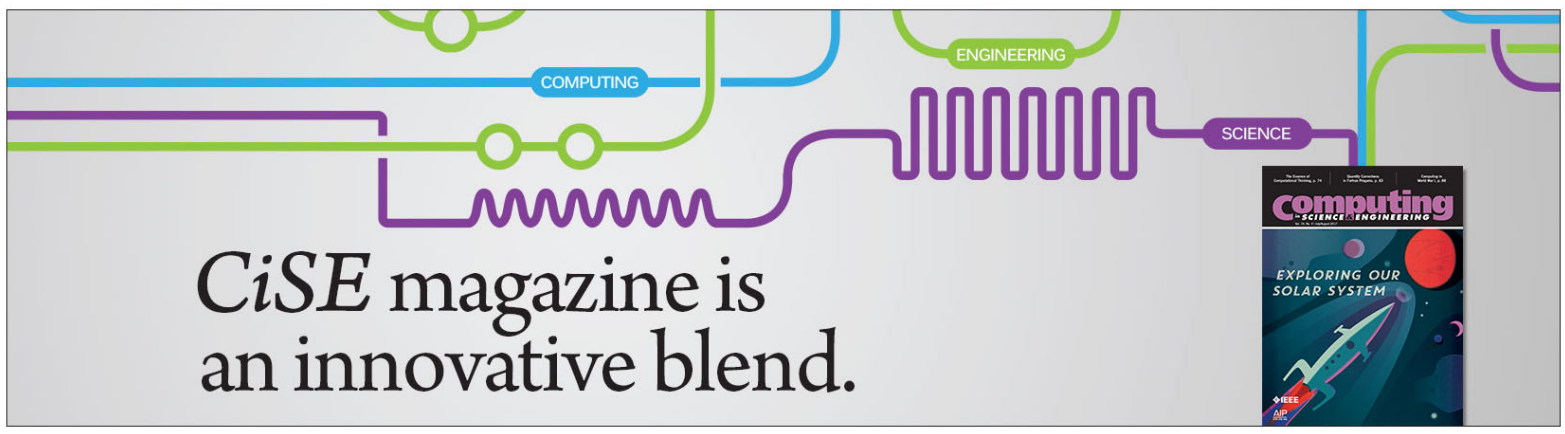




\title{
Demonstration of acoustic waveguiding and tight bending in phononic crystals
}

\author{
M. Ghasemi Baboly, ${ }^{1}$ A. Raza, ${ }^{2}$ J. Brady, ${ }^{2}$ C. M. Reinke, ${ }^{3}$ Z. C. Leseman, ${ }^{1}$ and I. El-Kady ${ }^{3, a)}$ \\ ${ }^{1}$ Department of Mechanical and Nuclear Engineering, Kansas State University, Manhattan, \\ Kansas 66506, USA \\ ${ }^{2}$ Department of Mechanical Engineering, University of New Mexico, Albuquerque, New Mexico 87131, USA \\ ${ }^{3}$ Department of Applied Photonic Microsystems, Sandia National Laboratories, Albuquerque, \\ New Mexico 87185, USA
}

(Received 27 August 2016; accepted 16 October 2016; published online 31 October 2016)

\begin{abstract}
The systematic design, fabrication, and characterization of an isolated, single-mode, $90^{\circ}$ bend phononic crystal $(\mathrm{PnC})$ waveguide are presented. A PnC consisting of a 2D square array of circular air holes in an aluminum substrate is used, and waveguides are created by introducing a line defect in the PnC lattice. A high transmission coefficient is observed $(-1 \mathrm{~dB})$ for the straight sections of the waveguide, and an overall $2.3 \mathrm{~dB}$ transmission loss is observed (a transmission coefficient of $76 \%$ ) for the $90^{\circ}$ bend. Further optimization of the structure may yield higher transmission efficiencies. This manuscript shows the complete design process for an engineered $90^{\circ}$ bend $\mathrm{PnC}$ waveguide from inception to experimental demonstration. Published by AIP Publishing.
\end{abstract}

[http://dx.doi.org/10.1063/1.4966463]

PnCs are periodic structures capable of selectively transmitting and controlling the propagation of acoustic waves. ${ }^{1}$ They can also exhibit bandgaps, where the propagation of such waves within a range of frequencies is forbidden in all directions. ${ }^{2}$ By introducing defects that distort the perfect array, isolated propagation modes can be created in the bandgap whereby one can realize PnC-enabled devices, such as resonators, ${ }^{3}$ waveguides, ${ }^{4} 90^{\circ}$ bends, ${ }^{5}$ acoustic isolators and filters, ${ }^{6}$ and even ultra-high Q cavities that operate at the material loss limit. $^{7} \mathrm{PnC}$ devices are especially useful in applications where power availability is an issue, because they are passive devices and thus require no power. This fact, combined with the compact miniature sizes of the devices, makes them attractive candidates for radio-frequency ( $R F$ ) applications, such as telecommunications, acoustic imaging, and focusing, and even mixed-physics devices such as optoacoustic modulators and opto-mechanical delay lines. ${ }^{8,9}$

Various materials have been used to design PnCs, including semiconductors, piezoelectrics, polymers, and metals, ${ }^{10-13}$ with each having various advantages and disadvantages relating to factors such as operating frequency, material loss, quality factor, and bandgap formation. Given that the manipulation of transmitted and/or blocked frequencies depends on the lattice symmetry and the shape and spacing of the inclusions, various types of lattices, such as square, ${ }^{14}$ triangular, honeycomb, and hexagonal, ${ }^{15}$ with various inclusions ranging from simple geometrical circular inclusions to fractal ${ }^{16}$ inclusions, have been studied using different numerical approaches, including plane-wave expansion, finite-difference time-domain, and finite-element method (FEM). ${ }^{17-19}$

As the miniaturization trend of RF acoustic circuits continues, there is an ever-increasing need for the efficient

\footnotetext{
${ }^{\text {a) }}$ Author to whom correspondence should be addressed. Electronic mail: ielkady@sandia.gov
}

guiding of acoustic signals to various extremities of chips. In the quest for chip real estate optimization, the guiding requirements often involve routing waves through a $90^{\circ}$ bend. In this communication, we focus on the design, fabrication, and characterization of $\mathrm{PnC}$ waveguides with the ultimate objective of realizing efficient guiding around a sharp corner. Kafesaki ${ }^{20}$ and Khelif ${ }^{21}$ showed theoretical demonstrations of straight waveguides using phononic crystals with minimal losses; however, there are hardly any experimental demonstrations of RF acoustic waveguiding through a bend. $^{22}$

Our PnC consists of a 2D square array of cylindrical air inclusions drilled into an aluminum slab. The inset of Fig. 1 shows a unit cell of the structure, where $a$ is the lattice constant, $t$ is the thickness of the slab, and $r$ is the radius of the air holes. To relax the fabrication constraints and provide rapid fabrication turnaround, a macro-scale lattice was realized where the above parameters $(a, t$, and $r)$ were set to $8 \mathrm{~mm}, 4 \mathrm{~mm}$, and $3.84 \mathrm{~mm}$, respectively, resulting in $r / a=0.48$ and $t / a=0.5$. A waveguide was subsequently constructed by introducing a line defect in an otherwise perfect array. It is important to realize that the scaling law of PnCs, demonstrated repeatedly throughout the literature, allows this design to be scaled down to micron length scales, thereby enabling the frequency of operation to be in the $\mathrm{MHz}^{23}$ or higher. The COMSOL finite element analysis package ${ }^{24}$ is used for the numerical analysis, and experimental measurements using piezoelectric transducers are conducted and compared to the theoretical findings. The following is a detailed description of the methodology employed.

A three-dimensional COMSOL Multiphysics model was created to determine the bandgap frequency range corresponding to the chosen PnC parameters. The dispersion relation of the first symmetric Brillouin zone of the structure was calculated using eigenfrequency analysis, and is shown in 


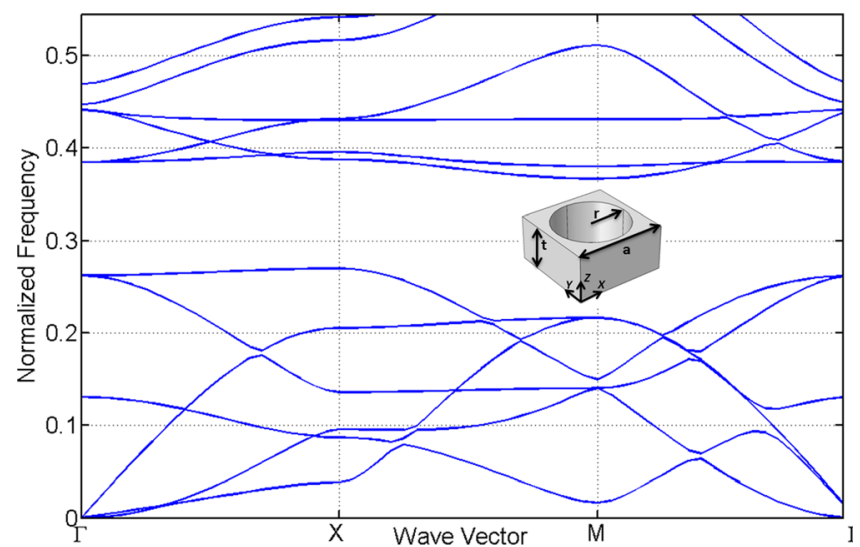

FIG. 1. Finite element analysis of the band structure of a bulk $2 \mathrm{D}$ square array of circular holes in Al-air phononic with $r / a=0.48$ and $t / a=0.5$. A unit cell of the structure is shown in the inset.

Fig. 1. The structure was simulated by utilizing a free boundary condition in the $z$-direction and periodic boundary conditions in the $\mathrm{x}$ - and $\mathrm{y}$-directions. All possible modes of propagation were considered, i.e., in-plane, out-of-plane transverse, and longitudinal modes. Examining the band diagram, it is clear that this arrangement provides a wide bandgap from 149 to $202 \mathrm{kHz}$ in which correspond to 0.27 to 0.366 in normalized frequency $\left(\Omega=\omega a / 2 \pi C_{t}\right.$, where $\omega$ is the angular frequency and $C_{t}$ is the transverse sound velocity) in which acoustic waves are not allowed to propagate in the crystal, resulting in 30\% gap-to-midgap ratio. This large bandgap allows for designing devices to guide mechanical vibrations over a wide frequency range.

The experimental setup consists of a function generator, digital oscilloscope, Butterworth filter, and piezoelectric transducers. Two wide-bandwidth piezoelectric transducers are mounted on either side of the devices to send and receive mechanical waves. Sinusoidal voltages are applied to the source transducer using the function generator, launching longitudinal and transverse waves. The waves propagate through the PnC device and are received by an identical transducer that is placed on the opposite side to sense the transmitted elastic waves and convert them back into a voltage signal that is detectable with the digital oscilloscope. Also, a 4th order Butterworth filter is placed after the receiving transducer, but before the oscilloscope, to reduce noise. The setup is first calibrated to adjust for ambient vibrations and impedance mismatch, then a reference signal is generated using an aluminum slab of the same geometrical envelope as the PnC devices. This reference signal is then used to normalize the transmission coefficients for all subsequent measurements of $\mathrm{PnC}$ devices. Two sets of experiments were performed, one with the perfect $\mathrm{PnC}$ lattice and one with the distorted lattice containing the line defect waveguide.

First, the perfect PnC was fabricated and tested; the results are shown in Fig. 2. It can be seen that the transmission coefficient drops between $\Omega=0.26$ and $\Omega=0.34$ in normalized frequency, indicating a wide and deep bandgap where incident waves are reflected. A frequency domain analysis was performed to reinforce the eigenfrequency analysis and calculate the transmission coefficient for the

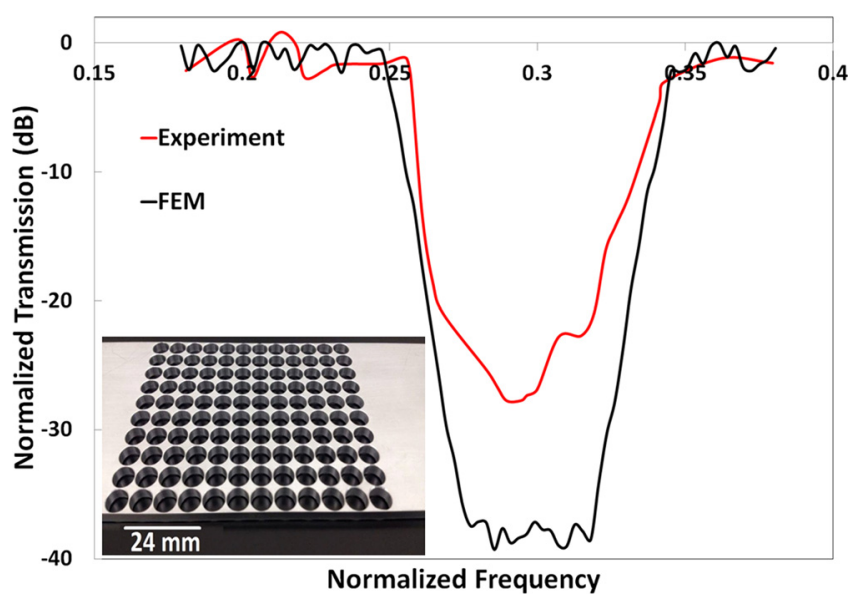

FIG. 2. Experimental (red) and numerical (black) transmission response of the Al-air PnC with $r / a=0.48$ and $t / a=0.5$. (Inset) Image of actual perfect PnC.

PnCs. ${ }^{25}$ The FEM results are in good agreement with experimental results, however FEM shows a deeper bandgap. This is expected, and can be attributed to insertion and material losses in the experiment which are not accurately represented in the simulation. In addition, the PnC lattice is assumed to be infinite in the FEM simulation, which results in a wider and deeper bandgap as compared to the finite-sized lattice that was actually fabricate and tested.

A straight waveguide is created by introducing a line defect in the crystal along the propagation direction. PnCs containing such line defects have been shown to guide elastic waves efficiently. ${ }^{26,27}$ The defect width, $w=(1+\alpha) a-2 r$, is defined as the separation distance between the edges of the two neighboring air holes on either side of the defect relative to the lattice constant $a$, where $\alpha$ is a scaling parameter. In this way, the width of the waveguide can be tuned with respect to lattice constant. For $\alpha=0$, we retrieve a perfect PnC (no defect) and for $\alpha=1$ we obtain the so-called W1 waveguide defined as a PnC plate where one row of holes is left out, i.e., $\mathrm{Al}$ remains in that row of holes. ${ }^{28}$

Figure 3 shows the band diagrams of the PnC samples in $\Gamma$-X direction (waveguiding direction) for various values of

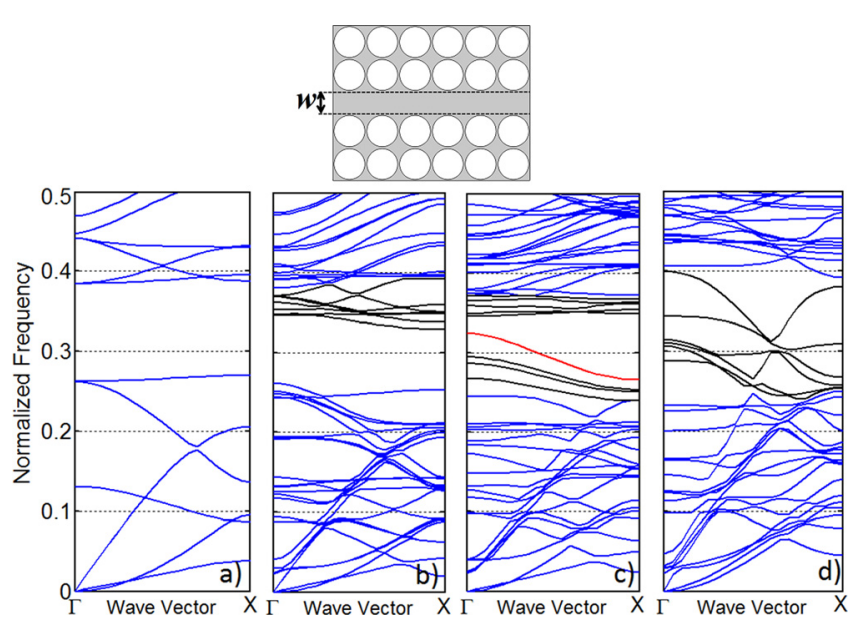

FIG. 3. Band structure along the $\Gamma-\mathrm{X}$ direction of phononic crystals with (a) $\alpha=0$ (perfect PnC) and also PnC devices containing a waveguide with (b) $\alpha=0.01$ (c) $\alpha=0.1$ (d) $\alpha=0.5$. (Top) schematic of the defect width (w). 
$\alpha$. Modes shown in black are those resulting from the introduction of the line defect. To ensure an efficient waveguide, a well-isolated defect mode is necessary. As can be seen, the choice of $\alpha=0.1$ produces a sufficiently isolated mode compared to the rest the cases and is shown in red. (Fig. 3(c)).

The $\alpha=0.1$ waveguide was then fabricated and tested. The experimental waveguide transmission response, plotted in Fig. 4, shows a passband inside the bandgap between $\Omega=0.25$ to 0.287 , centered at about 0.275 , showing $1 \mathrm{~dB}$ of transmission loss, which translates to $90 \%$ energy throughput. This is consistent with the FEM calculated band diagram of Fig. 3(c) as well as the calculated transmission shown in Fig. 4. Both the experiment and theory also confirm the existence of a complicated transmission band that can be attributed to the lower defect modes in Fig. 3(c). The frequency shift between the measured and calculated transmission spectra can be attributed to fabrication imperfections and the finiteness of the fabricated samples, as well as slight differences between the theoretical and actual material properties such as Young's modulus (E) and mass density $(\rho)$.

While both the upper isolated defect mode and the complex mix of the other defect modes are seen to transmit with high fidelity, it is imperative for guiding applications to be able to confine the guided energy along the waveguide axis. A zoom-in of the band diagram in Fig. 3(c) along with the associated mode shape at two frequencies is shown in Fig. 5. As can be seen, the lower frequency defect mode (black) is crossing bulk crystal modes and as a result is leaky, allowing the energy to travel through the whole crystal. Conversely, the higher-frequency mode (red) is well confined, enabling the guiding of waves only along the waveguide axis, and thus will form the basis of our $90^{\circ}$ bend design.

A bent waveguide was then designed and fabricated with $\alpha=0.1$. To create a contiguous $90^{\circ}$ waveguide bend, the lattice was slightly perturbed in the bend region by the introduction of reduced-size holes. The size of the small holes were chosen to keep the critical dimension, ${ }^{29}$ i.e., the minimum distance between inclusions, constant. In addition, a $45^{\circ}$ chamfer angle was introduced at the outer edge of the bend between the two perpendicular but otherwise identical line defects, see the insets of Fig. 6.

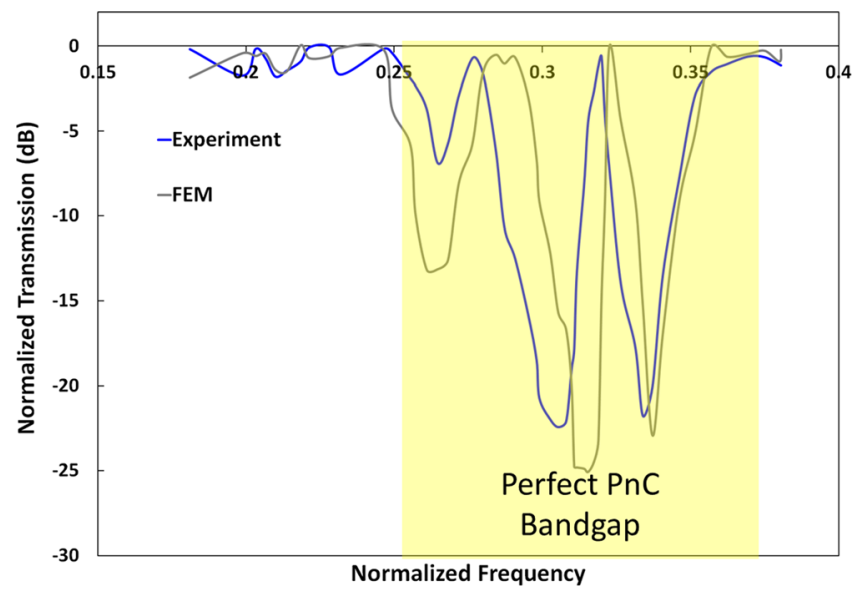

FIG. 4. Experimental (blue) and numerical (gray) transmission responses of the straight waveguide.

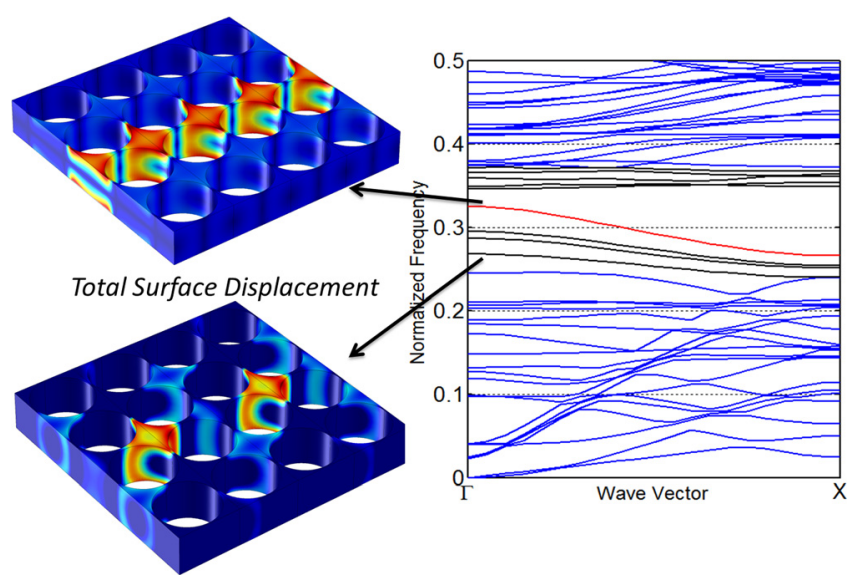

FIG. 5. Mode shape for guided modes: Inset shows the lower edge of the bandgap with the leaky mode that wave travels through the whole crystal and higher edge of the bandgap that has a confined guiding mode.

The experiment was repeated and the transmission spectrum then was extracted and plotted in Fig. 6. A transmission peak corresponding to the guided mode exists only in the defected PnC, as compared to the unperturbed lattice at $\Omega=0.32$, and corresponds well to the confined isolated mode at the same frequency in Fig. 4. The increase in the transmission close to the lower band edge, on the other hand, can be attributed to the complicated transmission of the lowerfrequency unconfined modes between $\Omega=0.25-0.285$, as discussed previously.

Finally, frequency domain FEM analysis was performed to provide insight into the efficiency of the bent waveguide and confinement of the mode along the defect. The left inset in Fig. 6 shows the numerical calculation of the propagation of the mode at $\Omega=0.26$ where the wave is not quite localized and mostly reflects back into the input, while the right inset of this figure shows a localized bent waveguide mode at $\Omega=0.32$ where the incident wave propagates along the first straight waveguide and couples efficiently in the perpendicular direction. Experimental transmission results indicate

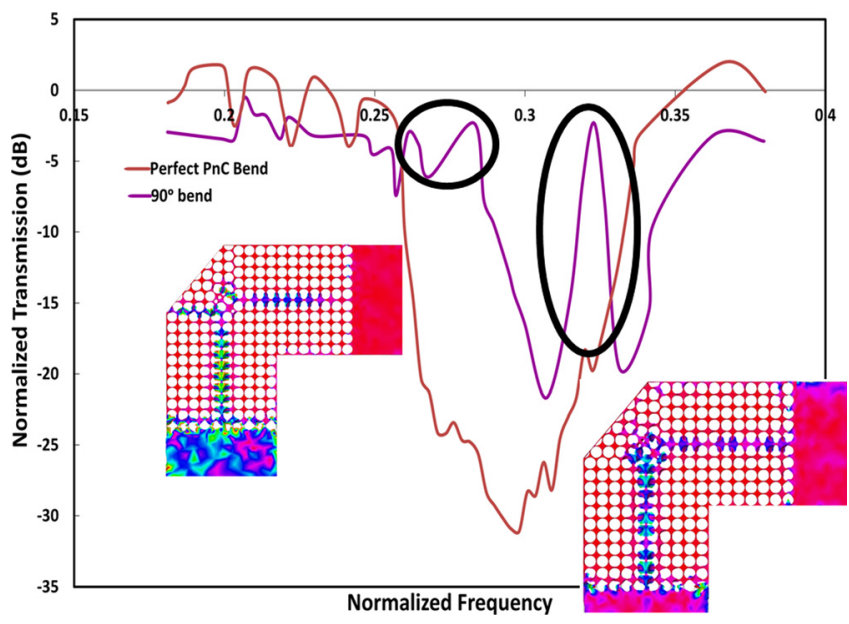

FIG. 6. Experimental results for the bent perfect PnC (red curve) and bent waveguide (purple curve) for the Al-air PnC. (Insets) To accommodate the $90^{\circ}$ bend, the lattice is disturbed and a few smaller inclusions that keep the same critical dimension are added. The total displacement field of the bent waveguide PnC for (left) leaky mode centered at $\Omega=0.26$ and (right) isolated mode at $\Omega=0.32$. 
this mode has a transmission coefficient of $-2.3 \mathrm{~dB}$ which corresponds to $76 \%$ energy transmission. Since the materials and dimensions as well as the $\mathrm{PnC}$ design here are different from other reported PnCs, only a qualitative comparison is possible. Nevertheless, comparing these results with the ones already published indicates that similar results could be achieved for different types of PnCs made of different materials. ${ }^{30,31}$

In conclusion, guiding and bending was demonstrated theoretically and experimentally in a planar phononic crystal composed of a square array of circular air cylinders in aluminum. We have shown that a confined guided mode can be realized by careful manipulation of the PnC lattice. Transmission as high as $90 \%$ was realized in the straight waveguide, and more than $76 \%$ in the $90^{\circ}$ bent case. The experimental results were in good agreement with the numerical analyses. This isolated, single-mode $90^{\circ}$ bend offers a paradigm based on bulk acoustic waves (BAW) for the design of next generation RF filters.

M.G.B., A.R., J.B., and Z.C.L. acknowledge the support from the National Science Foundation Division of CMMI under Award No. 1056077. The work was supported by Sandia National Laboratories, a multi-mission laboratory managed and operated by Sandia Corporation, and a wholly owned subsidiary of Lockheed Martin Corporation, for the U.S. Department of Energy's National Nuclear Security Administration under Contract No. DE-AC04-94AL85000.

${ }^{1}$ M. M. Sigalas, "Elastic wave band gaps and defect states in twodimensional composites," J. Acoust. Soc. Am. 101(3), 1256-1261 (1997).

${ }^{2}$ M. S. Kushwaha, P. Halevi, L. Dobrzynski, and B. Djafari-Rouhani, "Acoustic band structure of periodic elastic composites," Phys. Rev. Lett. 71(13), 2022-2025 (1993).

${ }^{3}$ S. Mohammadi, A. A. Eftekhar, W. D. Hunt, and A. Adibi, "High-Q micromechanical resonators in a two-dimensional phononic crystal slab," Appl. Phys. Lett. 94(5), 51906 (2009).

${ }^{4}$ N.-K. Kuo and G. Piazza, "Evidence of acoustic wave focusing in a microscale $630 \mathrm{MHz}$ aluminum nitride phononic crystal waveguide," in Proceedings of the IEEE International Frequency Control Symposium (2010), pp. 530-533.

${ }^{5}$ V. Liu and S. Fan, "Compact bends for multi-mode photonic crystal waveguides with high transmission and suppressed modal crosstalk," Opt. Express 21(7), 8069-8075 (2013).

${ }^{6} \mathrm{R}$. H. Olsson III and I. El-Kady, "Microfabricated phononic crystal devices and applications," Meas. Sci. Technol. 20(1), 12002 (2009).

${ }^{7}$ D. Goettler, M. Su, Z. Leseman, Y. Soliman, R. Olsson, and I. El-Kady, "Realizing the frequency quality factor product limit in silicon via compact phononic crystal resonators," J. Appl. Phys. 108(8), 84505 (2010).

${ }^{8}$ H. Shin, J. A. Cox, R. Jarecki, A. Starbuck, Z. Wang, and P. T. Rakich, "Control of coherent information via on-chip photonic-phononic emitter-receivers," Nat. Commun. 6, 2-9 (2015).

${ }^{9}$ S. Alaie, M. Hossein-zadeh, S. Member, M. G. Baboly, M. Zamani, and Z. C. Leseman, "Enhancing mechanical quality factors of micro-toroidal optomechanical resonators using phononic crystals," JMEMS 25(2), 311-319 (2016).

${ }^{10}$ Y. M. Soliman, M. F. Su, Z. C. Leseman, C. M. Reinke, I. El-Kady, and R. H. Olsson, "Phononic crystals operating in the gigahertz range with extremely wide band gaps," Appl. Phys. Lett. 97(19), 193502 (2010).
${ }^{11}$ N.-K. Kuo, C. Zuo, and G. Piazza, "Microscale inverse acoustic band gap structure in aluminum nitride," Appl. Phys. Lett. 95(9), 93501 (2009).

${ }^{12}$ Y. Tanaka and S. Tamura, "Surface acoustic waves in two-dimensional periodic elastic structures," Phys. Rev. B 58(12), 7958-7965 (1998).

${ }^{13}$ B. Jiang, W. Zhou, A. Liu, W. Chen, and W. Zheng, "Improved combined wave number eigenvalue equations method for band structure calculations of metal photonic crystal," Opt. Commun. 285(7), 1859-1863 (2012).

${ }^{14}$ M. Ghasemi Baboly, S. Alaie, C. M. Reinke, I. F. El-Kady, and Z. C. Leseman, "Ultra-high frequency, high Q/volume micromechanical resonators in a planar AlN phononic crystal Ultra-high frequency, high Q/volume micromechanical resonators in a planar AlN phononic crystal," J. Appl. Phys. 120(3), 034502 (2016).

${ }^{15}$ S. Mohammadi, A. A. Eftekhar, A. Khelif, W. D. Hunt, and A. Adibi, "Evidence of large high frequency complete phononic band gaps in silicon phononic crystal plates," Appl. Phys. Lett. 92(22), 221905 (2008).

${ }^{16} \mathrm{~N}$.-K. Kuo and G. Piazza, "Ultra high frequency air/aluminum nitride fractal phononic crystals," in Proceedings of the Joint Conference of the IEEE International Frequency Control and the European Frequency and Time Forum (2011), pp. 1-4.

${ }^{17}$ M. G. Baboly, Y. Soliman, M. F. Su, C. M. Reinke, Z. C. Leseman, and I. El-Kady, "Enhanced plane wave expansion analysis for the band structure of bulk modes in two-dimensional high-contrast solid-solid phononic crystals," Photonics Nanostruct. -Fundam. Appl. 12(5), 487-492 (2014).

${ }^{18}$ J.-H. Sun and T.-T. Wu, "Propagation of surface acoustic waves through sharply bent two-dimensional phononic crystal waveguides using a finite-difference time-domain method," Phys. Rev. B 74(17), 174305 (2006).

${ }^{19}$ M. Ghasemi Baboly, M. F. Su, C. M. Reinke, S. Alaie, D. F. Goettler, I. El-Kady, and Z. C. Leseman, "The effect of stiffness and mass on coupled oscillations in a phononic crystal," AIP Adv. 3(11), 112121 (2013).

${ }^{20}$ M. Kafesaki, M. M. Sigalas, and N. García, "Frequency modulation in the transmittivity of wave guides in elastic-wave band-gap materials," Phys. Rev. Lett. 85(19), 4044-4047 (2000).

${ }^{21}$ A. Khelif, J. O. Vasseur, P. A. Deymier, P. Lambin, and L. Dobrzynski, "Transmittivity through straight and stublike waveguides in a twodimensional phononic crystal," Phys. Rev. B 65, 174308 (2002).

${ }^{22}$ A. Khelif, A. Choujaa, S. Benchabane, B. Djafari-Rouhani, and V. Laude, "Guiding and bending of acoustic waves in highly confined phononic crystal waveguides," Appl. Phys. Lett. 84(22), 4400 (2004).

${ }^{23}$ R. Ramprasad and N. Shi, "Scalability of phononic crystal heterostructures," Appl. Phys. Lett. 87, 111101 (2005).

${ }^{24} \mathrm{See}$ http://www.comsol.com/ for "COMSOL is a multiphysics software package for performing finite-element-method (FEM) simulations."

${ }^{25}$ S. Alaie, M. F. Su, D. F. Goettler, I. El-Kady, and Z. Leseman, "Effects of flexural and extensional excitation modes on the transmission spectrum of phononic crystals operating at gigahertz frequencies," J. Appl. Phys. 113(10), 103513 (2013).

${ }^{26}$ Y. Pennec, B. Djafari-Rouhani, J. O. Vasseur, A. Khelif, and P. A. Deymier, "Tunable filtering and demultiplexing in phononic crystals with hollow cylinders," Phys. Rev. E 69, 046608 (2004).

${ }^{27}$ T. Miyashita, "Acoustic defect-mode waveguides fabricated in sonic crystal : Numerical analyses by elastic finite-difference time-domain method," Jpn. J. Appl. Phys., Part 1 45(5B), 4440-4447 (2006).

${ }^{28}$ J. Vasseur, P. Deymier, B. Djafari-Rouhani, Y. Pennec, and A.-C. HladkyHennion, "Absolute forbidden bands and waveguiding in two-dimensional phononic crystal plates," Phys. Rev. B 77(8), 85415 (2008).

${ }^{29}$ S. Alaie, D. F. Goettler, M. Su, Z. C. Leseman, C. M. Reinke, and I. Elkady, "Thermal transport in phononic crystals and the observation of coherent phonon scattering at room temperature," Nat. Commun. 6, 7228 (2015).

${ }^{30}$ A. Khelif, A. Choujaa, M. Wilm, S. Ballandras, and V. Laude, "Trapping and guiding of acoustic waves by defect modes in a full-band-gap ultrasonic crystal," Phys. Rev. B 68, 214301 (2003).

${ }^{31}$ T. Miyashita, W. Sato, Y. Nakaso, and R. Mukuda, "Experimental studies on two-dimensional defect-mode waveguides in a sonic/phononic crystal," Jpn. J. Appl. Phys., Part 1 46(7B), 4684-4687 (2007). 